\title{
Separating an allele associated with late flowering and slow maturation of Arabidopsis thaliana from population structure
}

Yanjun $\operatorname{Zan}^{1 \odot}$, Xiao Feng ${ }^{2,3 \odot}$, Zheng Ning ${ }^{3}$, Weilin $\mathrm{Xu}^{3,4}$, Qianhui $\mathrm{Wan}^{3,5}$, Dongyu Zeng ${ }^{6}$, Ziyi Zeng ${ }^{7}$, Yang Liu ${ }^{*}$, Xia Shen ${ }^{3,8^{*}}$

1 Department of Medical Biochemistry and Microbiology, Uppsala University, Uppsala, Sweden

2 State Key Laboratory of Biocontrol, Guangdong Provincial Key Laboratory of Plant Resources, Key Laboratory of Biodiversity Dynamics and Conservation of Guangdong Higher Education Institutes, School of Life Sciences, Sun Yat-Sen University, Guangzhou, China

3 Department of Medical Epidemiology and Biostatistics, Karolinska Institutet, Stockholm, Sweden

4 Department of Molecular Biology and Genetics, Cornell University, Ithaca, NY, USA 5 Department of Mathematics, University of Wisconsin-Madison, Madison, Wisconsin, USA

6 School of Life Sciences, Sun Yat-Sen University, Guangzhou, China

7 School of Engineering, Sun Yat-Sen University, Guangzhou, China

8 Center for Global Health Research, Usher Institute of Population Health Sciences and Informatics, University of Edinburgh, Edinburgh, Scotland, UK

These authors contributed equally to this work.

* Correspondence to xia.shen@ed.ac.uk (X.S.) or liuy353@mail.sysu.edu.cn (Y.L.)

\section{Abstract}

Genome-wide association analysis is a powerful tool to identify genomic loci underlying complex traits. However, the application in natural populations comes with challenges, especially power loss due to population stratification. Here, we introduce a bivariate analysis approach to a GWAS dataset of Arabidopsis thaliana. A common allele, 
strongly confounded with population structure, is discovered to be associated with late flowering and slow maturation of the plant. The discovered genetic effect on flowering time is further replicated in independent datasets. Using Mendelian randomization analysis based on summary statistics from our GWAS and expression QTL scans, we predicted and replicated a candidate gene AT1G11560 that potentially causes this association. Further analysis indicates that this locus is co-selected with flowering-time-related genes. We demonstrate the efficiency of multi-phenotype analysis to uncover hidden genetic loci masked by population structure. The discovered pleiotropic genotype-phenotype map provides new insights into understanding the genetic correlation of complex traits.

\section{Author Summary}

Joint-analyzing multiple phenotypes is of increasing interest in this post-GWAS era, because of its potential power to reveal more discoveries and its potential insights into pleiotropic genetic architecture. Here, using publicly available $A$. thaliana data, we provide a "textbook" empirical evidence showing how a novel allele, highly confounded with population structure but carries a large genetic effect, can be detected via a double-trait analysis. The allele postpones the flowering time and maturation endpoint of the plant at the same time. The discovered genetic effect can be replicated. We illustrate the bivariate genotype-phenotype map that produces such statistical power. Combining with gene expression genomic scans, we also predict candidate genes using summary-level Mendelian randomization analysis. The results indicate that multi-phenotype analysis is a powerful and reliable strategy to uncover additional value in the established GWAS data.

\section{Introduction}

Evolution has resulted in the speciation and adaptation of various organisms. Although natural selection applies to all kinds of species, the resulted natural population structures have dramatic difference. Especially, due to their lack of mobility, plants, comparing to humans and most animals, have established much stronger population 
structure adaptive to specific climate conditions (Ch. 11 in [1]). This makes it difficult, for instance in modern genomic studies, to distinguish genotypic effects on plants' phenotypes from geographical stratification [2].

Fast-developing genotyping techniques have made genome-wide association study (GWAS) one of the most useful approaches for discovering genomic loci that regulate phenotypes in various organisms 2, 4]. In human GWAS, we learnt that most of the discovered loci associated with complex traits or disease have very small effects [5]. The detected single nucleotide polymorphisms (SNPs) need to have sufficiently high minor allele frequencies (MAFs) for the statistical tests to gain enough power, while high-MAF variants tend to have small effects on the studied phenotypes as these variants were under weak selection pressure. Alleles that have high penetrance on a phenotype are normally under strong selection, resulting in low MAFs of the corresponding SNPs. Thus, a major challenge in human GWAS appears to be the trade-off between statistical power and the effect size of the variant to detect 68 .

Although similar trade-off also applies to GWAS in plant populations, e.g. in the natural population of Arabidopsis thaliana, in terms of discovery power, the major challenge is different. As each individual plant accession is sampled from a specific geographical location in the world, accessions with different genotypes normally have much greater phenotypic differences compared to those in humans. It appears that the genome can explain a large proportion of variation in the plant phenotype, however, the population structure in nature makes such a genomic effect heavily confounded with the environmental effect due to geographical stratification. Therefore, there can be a number of alleles, who have large genetic effects on a certain phenotype, but masked by the population structure.

As a community based effort, over 1000 natural $A$. thaliana accessions have been collected from worldwide geographical locations 9,10$]$. Most of those plants have been sequenced for genome, transcriptome, and even methylome, and these datasets have been made publicly available for worldwide researchers. Many accessions in this collection have been phenotyped for developmental, metabolic, inomics, stress resistance traits 2], and more and more phenotypes are gradually releasing. Previous analysis in those datasets have revealed substantial connections between genotypic and phenotypic variations in this species. The application of association mapping have provided insights 
to the genetic basis of complex traits 2, 11, 12, adaptation [13] and evolutionary process. Nevertheless, many essential genotype-phenotype links are still difficult to establish based on the current GWAS data, due to the substantial population stratification highly correlated with the sampling origins of the plants. Therefore, novel powerful analyses are required to further uncover hidden genetic regulation.

Based on publicly available $A$. thaliana datasets $[2,9,10,14$, here, we aim to use a bivariate analysis method that combines the discovery power of two correlated phenotypes [15], in order to map novel pleiotropic loci that simultaneously regulate both traits. We interpret the statistical significance with a double-trait genotype-phenotype map. We try to replicate and in silico functionally investigate the candidate genes that may drive such associations.

\section{RESULTS}

\section{Bivariate genomic scan identifies a hidden locus simultaneously associated with flowering and maturation periods}

We re-analyzed a public dataset of a natural $A$. thaliana collection, where 43 developmental phenotypes and 23 flowering-time-related phenotypes were previously published [2]. The number of accessions with measured phenotypes varies from 93 to 193 with a median of 147 (Table S1). We first excluded all variants with minor allele frequencies (MAF) less than 0.1 and performed single-trait GWA analysis for all these traits based on a linear mixed model, so that the confounded genetic effects due to population stratification is adjusted. We then applied our recently developed multi-trait GWAS method [15] to all pairwise combination of the phenotypes (Materials \&

Methods). One novel locus, in one of the pairwise test, reached the most stringent $5 \%$ Bonferroni-corrected genome-wide significance threshold for the 2,145 pairs of traits and 173,220 variants, i.e. $p<1.35 \times 10^{-10}$ (Table 1 , Figure 1a). This signal also reaches single-trait genome-wide significance in other six pairs of traits highly correlated with the top pair (Figure S1), without Bonferroni-correction for the number of tested trait pairs (Table 1, Figure S3-S8).

For the most significant trait combination, 2W (days to flowering time under long 
day with vernalized for 2 weeks) and MT GH (maturation period), the linkage

67 disequilibrium (LD) block of this locus (LD $r>0.7$ ) covers about a $260 \mathrm{~kb}$ interval on chromosome 1 , with a top variant at 3,906,923 bp (double-trait $p=9.9 \times 10^{-12}$, Figure 1b, Table 1). The detected locus shows joint effects on flowering and maturation, where the effect on flowering time $(2 \mathrm{~W})$ is notably large (15.3 days), and that on maturation period (MT GH) is 2.5 days (Table 1 ). These correspond to narrow-sense heritability values of $24 \%$ and $10 \%$ of the two phenotypes, respectively.

[TABLE 1 ABOUT HERE]

[FIGURE 1 ABOUT HERE]

\section{Double-trait analysis is sufficiently powerful to overcome the confounding population structure}

The detected joint-effect locus was missed in the corresponding single-trait GWA analysis of $2 \mathrm{~W}$ (effect $=15.3, p=2.26 \times 10^{-5}$ after correcting for population stratification) and that of MT GH (effect $\left.=2.5, p=3.70 \times 10^{-5}\right)$. Notably, this locus was not even detectable at the genome-wide significance level in a much larger population of more than 1,000 A. thaliana accessions [9,10] due to its severe confounding with the natural population structure. The statistical significance can only be identified when considering the joint distribution of the bivariate statistic. According to the genome-wide Z-scores (student t-statistics), these two phenotypes are negatively correlated, as the plant's lifespan is relatively stable (estimated and observed phenotypic correlation $=-0.55$ and -0.68 , respectively). However, the observed effects on the two traits are both substantially positive, showing sufficient deviation from the joint distribution that led to bivariate statistical significance (Figure 2).

[FIGURE 2 ABOUT HERE]

The strong confounding with the population structure can also be visualized by the allele frequency distribution of the top associated SNP across different $A$. thaliana sub-populations based on the genome re-sequencing data from the A. thaliana 1001-genomes project [9] (Figure 3). The sub-populations were divided by admixture analysis using ADMIXTURE 9,16 . The plus allele increasing flowering time was 
predominantly found in Sweden and almost fixed in the Northern Sweden population

(Figure 3b; allele frequency $=0.97$ in Northern Sweden and 0.51 in Southern Sweden).

Overall, the phenotype, e.g. flowering time at $10^{\circ} \mathrm{C}$, highly correlates with the

frequency of the plus allele (Figure 3). The genotype at this locus follows a latitude

decline, where the northern accessions are enriched with the plus allele and the southern accessions are enriched with the minus allele (Figure 3). This spatially imbalanced enrichment shows strong confounding with the population structure, which is why standard single-trait GWAS loses power substantially.

[FIGURE 3 ABOUT HERE]

96

97

98

99

100

101

102

103

104

\section{Replication of the detected genetic effect on flowering time}

Although we are lack of an independent dataset of $A$. thaliana maturation duration to replicate the bivariate statistical test, datasets containing additional independent $A$.

thaliana flowering time measurements are available. We downloaded a flowering time

GWAS dataset measured in 1,135 natural accessions from the 1001-genomes project

collection [9] and performed a single-trait association analysis of our discovered top SNP

with linear mixed model correction for the population structure. The genetic effect was significantly replicated for flowering time at $10{ }^{\circ} \mathrm{C}($ effect $=1.7$ days, $p=0.037)$ and

flowering time at $16{ }^{\circ} \mathrm{C}$ (effect $=3.6$ days, $\left.p=0.003\right)$. The effects on flowering time in the replication sample appear to be smaller than in the discovery population, possibly due to Winner's curse in the discovery phase.

We also screened literature for conventional quantitative trait loci (QTL) studies in intercrosses using natural $A$. thaliana accessions. Our detected signal is underneath a reported QTL peak for flowering time from an intercross between a Swedish and an Italian accession 17] (Figure S2). This, together with the replication above, justifies the detected association. Although the discovered genetic effect on maturation period is not directly replicated, the effect does exists when the effect on flowering is justified, as the pleiotropic signal must be driven by both phenotypes. 


\section{Prediction and replication of candidate genes using summary-level Mendelian randomization}

As a community-based effort, all the natural A. thaliana accessions from the 1001-genomes project were measured for their transcriptome 9,10 . Such a public gene expression dataset allows us to predict candidate genes underlying the association signal. We extracted the expression levels of 19 genes within a $\pm 20 \mathrm{~kb}$ window around the top associated SNP using RNA-seq gene expression measurements from 140 accessions [14]. Among these, the distributions of 14 gene expression phenotypes significantly deviate from normality (Kolmogorov-Smirnov test statistic $>0.8$ ), and these genes were filtered out due to potential unreliable measurements [18. The remaining 5 genes were passed onto eQTL mapping at the discovered locus (Materials \& Methods).

Based on the locus-specific eQTL mapping summary statistics, we applied the recently developed Summary-level Mendelian Randomization (SMR) method 19 to predict potential candidate genes among these five genes. The analysis integrates summary association statistics from GWAS and eQTL scan to predict functional candidate genes using multiple-instrument Mendelian randomization [20], where the complementary HEterogeneity In Dependent Instruments (HEIDI) test checks that the gene expression and flowering time share the same underlying causal variant. One significant candidate AT1G11560 was detected after Bonferroni correction for five tests (Figure 4, Table 2). This candidate gene prediction result was also replicated using an independent eQTL mapping dataset 10 .

[TABLE 2 ABOUT HERE]

[FIGURE 4 ABOUT HERE]

\section{Indication of co-selection with genes in flowering-related pathways}

As flowering time is a well-known polygenic trait, we expect multiple loci to be involved and possibly co-selected as a result of parallel evolution. Therefore, we explored the evidence of co-selection by associating the expression values of 288 known genes in flowering-time-related pathways and 1 gene in the maturation related pathway with our top SNP using transcriptome data from 648 A. thaliana accessions [9] (Materials \&
125 126 127 128 129 
Methods). In total, six genes (NF-YA8, AT5G53360, SPL15, AGL42, FLC, AGL20)

152

were associated with our top SNP (false discovery rate $<0.05$ ), where, conservatively,

four genes (AT5G53360, AGL42, FLC, AGL20) were replicated after Bonferroni

correction for six tests using data from an independent collection of 140 A. thaliana 14

(Table 3). This indicates that co-selected genes in multiple pathways determine the

flowering time variation in nature, and our detected locus contributes to a part of that

[TABLE 3 ABOUT HERE]

\section{DISCUSSION}

A serious issue of GWAS in natural population is the confounding between true underlying genetic effects and the population structure, which can lead to spurious associations between genotypes and phenotypes if population stratification is not properly adjusted $[6] 8$. Incorporation of the random polygenic effect using linear mixed models can effectively control the population structure, but such correction often compromises the true signals. Here, we applied a bivariate analysis to a classic dataset and successfully separated a locus from strong population structure. The detected allele is associated with late flowering and slow maturation of $A$. thaliana, which was corrected away by the linear mixed model in standard single-trait analysis. The replication of the genetic effect on flowering time in an old intercross linkage analysis and another independent dataset improves the confidence of this association. The discovered association is a typical example that jointly modeling phenotypes that share genetic basis can boost discovery power and reveal pleiotropic genotype-phenotype map at the same time.

Together with our recent application of multivariate analysis in human isolated populations [15, the results further indicate that multi-phenotype analysis is an effective approach to detect hidden loci that are lack of discovery power in single-phenotype analysis thus is worth testing in broader applications. Multivariate analysis appears to have the greatest power when the locus-specific genetic correlation does not agree with the natural phenotypic correlation. For instance, like the discovery here, for two traits that are negatively correlated, loci that generate positive genetic correlation between the traits tend to have good chance to be detected in a joint analysis.
153 
In GWAS, phenotypes are usually chosen based on morphological, physiological or economical features. Those features are usually feasible and simple to quantify; however, they might not be directly representative for the underlying genetic or biological factor that we try to detect. Fortunately, a certain degree of biological pathway sharing among complex traits is common, i.e. pleiotropy 21. Nowadays, it is very common that multiple phenotypes are measured for same individuals in many GWAS datasets, especially in omics study where thousands of phenotypes are measured. Instead of focusing on one phenotype at a time, it is of essential value to jointly model multiple phenotypes, attempting to detect pleiotropic loci that affect multiple traits with biological relevance.

In this study, all the pairs of traits that are associated with the detected locus contain at least one flowering-time trait, and nearly all of them have maturation duration involved. Detection of the novel locus in a bivariate analysis indicates shared genetic basis for the two types of developmental traits, which measure the lengths of two important period during the plant's life time. By integrating the expression level information and GWAS result using SMR/HEIDI test, we were able to predict candidate genes in this region. However, further work beyond the scope of this paper is still required to establish the molecular biological basis underlying the replicate association.

Many genetic variants affecting flowering time have been mapped and many genes promoting flowering times have been well characterized using standard lab accession, Col-0 [22]. Unlike simple traits, where only one or a few alleles are driving the trait's variation, there are many more variants throughout the genome that contribute to the variation of flowering time. The associations between our top SNP and the expression of many flowering-time-related genes serve as evidence of co-selection or parallel adaptation.

In conclusion, our study demonstrates the efficiency of joint modeling multiple-phenotypes which overcomes severe power loss due to population stratification in association studies. We discover and replicate a pleiotropic allele that regulate flowering and maturation periods simultaneously, providing novel insights in understanding the plant's development over life time. By integrating gene expression information with the GWAS results, we predict a functional candidate underneath the associated genomic region. Analysis of gene expression with other flowering-time-related 
genes show evidence of co-selection of the predicted candidate with many genes in

flowering-time pathways. We encourage wider applications of such a multivariate

framework in future analyses of genomic data.

\section{Acknowledgements}

X.S. was in receipt of a Swedish Research Council (VR) grant (No. 537-2014-371).

International collaboration within this work was partly supported by the Swedish

Foundation for International Cooperation in Research and Higher Education (STINT)

initiation grant to X.S. (No. IB2015-6000) and Karolinska Institutet travel grant (No.

2017-00534). The funders had no role in study design, data collection and analysis, decision to publish, or preparation of the manuscript.

\section{Author contributions}

X.S. initiated and coordinated the study. Y.Z. and X.F. performed the main data analysis. Z.N. and X.S. contributed to statistical modeling and interpretation. W.X., Q.W., D.Z. and Z.Z. contributed to data processing. Y.Z., X.F. and X.S. wrote the manuscript. Y.L. and X.S. supervised the study.

\section{Competing interests statement}

The authors have declared that no competing interests exist. 


\section{Tables}

Table 1: Discovery and replication analyses results for the novel pleiotropic ${ }_{232}$

locus. Reported association statistics are for the top variant at the locus for each pair $\quad 233$ of traits. ${ }^{1}$ LD: Days to flowering time under Long Day. ${ }^{2} 0 \mathrm{~W}$ : Days to flowering time $\quad{ }^{234}$ under long day without vernalization. ${ }^{3} 2 \mathrm{~W}$ : Days to flowering time under long day with $\quad{ }_{235}$ vernalized for 2 weeks at $5{ }^{\circ} \mathrm{C}, 8 \mathrm{hrs}$ daylight. ${ }^{4} 4 \mathrm{~W}$ : Days to flowering time under long $\quad{ }_{236}$ day with vernalized for 4 weeks at $5^{\circ} \mathrm{C}, 8 \mathrm{hrs}$ daylight. ${ }^{5} \mathrm{OW}$ GH FT: Days to flowering ${ }^{237}$ time (greenhouse). ${ }^{6} \mathrm{FT}$ GH: Days to flowering (greenhouse). ${ }^{7} \mathrm{MT}$ GH: Maturation $\quad{ }^{238}$ period (greenhouse), $20^{\circ} \mathrm{C}, 16 \mathrm{hrs}$ daylight. ${ }^{8} \mathrm{RP} \mathrm{GH}$ : Reproduction period (greenhouse), ${ }^{239}$ $20^{\circ} \mathrm{C}, 16$ hrs daylight. ${ }^{9} \mathrm{RA}$ : Reference allele. ${ }^{10} \mathrm{EA}$ : Effect allele. ${ }^{11} \mathrm{MAF}$ : Minor allele $\quad{ }_{240}$ frequency. ${ }^{12}$ Correlation refers to observed phenotypic correlation. ${ }^{13} \mathrm{FT}$ : Flowering $\quad{ }^{241}$ time.

\begin{tabular}{|c|c|c|c|c|c|c|c|c|c|c|c|}
\hline \multicolumn{12}{|c|}{ Double-trait Analysis } \\
\hline \multicolumn{2}{|l|}{ Trait 1} & \multicolumn{2}{|c|}{ Trait 2} & \multirow{2}{*}{$\frac{\mathrm{Chr}}{1}$} & Position & $\mathrm{RA}^{9}$ & $\mathrm{EA}^{10}$ & $\mathrm{MAF}^{11}$ & \multicolumn{2}{|c|}{$P$} & Correlation $^{12}$ \\
\hline \multicolumn{2}{|l|}{$\mathrm{LD}^{1}$} & \multicolumn{2}{|c|}{$\mathrm{MT} \mathrm{GH}^{7}$} & & 3895353 & $\mathrm{C}$ & $\mathrm{T}$ & 0.20 & \multicolumn{2}{|c|}{$6.3 \times 10^{-9}$} & -0.39 \\
\hline \multicolumn{2}{|l|}{$0 \mathrm{~W}^{2}$} & \multicolumn{2}{|c|}{$\mathrm{MT} \mathrm{GH}^{7}$} & 1 & 3896072 & G & $\mathrm{T}$ & 0.20 & \multicolumn{2}{|c|}{$8.4 \times 10^{-9}$} & -0.58 \\
\hline \multicolumn{2}{|l|}{$2 \mathrm{~W}^{3}$} & \multicolumn{2}{|c|}{$\mathrm{MT} \mathrm{GH}{ }^{7}$} & 1 & 3906923 & $\mathrm{~T}$ & $\mathrm{C}$ & 0.22 & \multicolumn{2}{|c|}{$9.9 \times 10^{-12}$} & $-0.68^{244}$ \\
\hline \multicolumn{2}{|l|}{$2 \mathrm{~W}^{3}$} & \multicolumn{2}{|c|}{$\mathrm{RP} \mathrm{GH}^{8}$} & 1 & 3978064 & A & $\mathrm{C}$ & 0.27 & $1.3 \times$ & $10^{-8}$ & -0.17 \\
\hline $4 \mathrm{~W}^{4}$ & & MT & $\mathrm{GH}^{7}$ & 1 & 3906923 & $\mathrm{~T}$ & $\mathrm{C}$ & 0.22 & $3.1 \times$ & $10^{-9}$ & -0.64 \\
\hline oW GH & $\mathrm{FT}^{5}$ & MT & $\mathrm{GH}^{7}$ & 1 & 3906923 & $\mathrm{~T}$ & $\mathrm{C}$ & 0.22 & $1.8 \times$ & $10^{-8}$ & -0.36 \\
\hline FT GH & & MT & $\mathrm{GH}^{7}$ & 1 & 3896072 & G & $\mathrm{T}$ & 0.20 & $1.5 \times$ & $10^{-8}$ & -0.60 \\
\hline Single-t & rait $A_{1}$ & alysi: & & & & & & & Repli & cation & \\
\hline & Trait & & & & Trait 2 & & & $\mathrm{~T}^{13} 10^{\mathrm{C}}$ & & & $1316^{\circ} \mathrm{C}$ \\
\hline Effect & $P$ & & $h^{2}$ & Effect & $P$ & $h^{2}$ & Effect & & $P$ & Effect & $P$ \\
\hline 33.5 & $5.6 \times$ & $0^{-6}$ & 0.22 & 2.42 & $6.0 \times 10^{-4}$ & 0.0 & 1.95 & 2.3 & $10^{-2}$ & 3.96 & $1.5 \times 10^{-3}$ \\
\hline 17.3 & $1.6 \times$ & $0^{-4}$ & 0.17 & 2.59 & $2.1 \times 10^{-4}$ & $\begin{array}{ll}-4 & 0.0\end{array}$ & 1.95 & $2.3>$ & $10^{-2}$ & 3.96 & $1.5 \times 10^{-3}$ \\
\hline 15.3 & $2.3 \times$ & $0^{-5}$ & 0.24 & 2.47 & $3.7 \times 10^{-5}$ & 0.1 & 1.72 & 3. & $0^{-2}$ & 3.56 & $3.0 \times 10^{-3}$ \\
\hline 19.7 & $6.8 \times$ & $0^{-7}$ & 0.26 & 2.65 & $1.6 \times 10^{-3}$ & 0.0 & 1.57 & $5.6>$ & $10^{-2}$ & 2.57 & $3.4 \times 10^{-2}$ \\
\hline 11.6 & $1.7 \times$ & $0^{-3}$ & 0.16 & 2.47 & $3.7 \times 10^{-5}$ & 0.1 & 1.72 & $3.7>$ & $10^{-2}$ & 3.56 & $3.0 \times 10^{-3}$ \\
\hline 25.8 & $3.8 \times$ & $0^{-5}$ & 0.21 & 2.47 & $3.7 \times 10^{-5}$ & 0.1 & 1.72 & $3.7>$ & $10^{-2}$ & 3.56 & $3.0 \times 10^{-3}$ \\
\hline 14.9 & $1.8 \times$ & $0^{-3}$ & 0.11 & 2.59 & $2.1 \times 10^{-4}$ & $\begin{array}{ll}-4 & 0.0\end{array}$ & 1.95 & $2.3 x$ & $10^{-2}$ & 3.96 & $1.5 \times 10^{-3}$ \\
\hline
\end{tabular}


Table 2: Summary of the SMR/HEIDI analysis results. ${ }^{1}$ Top SNP: The top $\quad{ }^{247}$ SNP in expression QTL analysis. ${ }^{2} \mathrm{MAF}$ : Minor allele frequency of the top associated SNP. ${ }^{3} P_{\mathrm{SMR}}$ : p-value from SMR using a collection of $140 \mathrm{~A}$. thaliana accessions.

${ }^{4} P_{\text {HEIDI }}$ : p-value from HEIDI test using a collection of $140 \mathrm{~A}$. thaliana. ${ }^{5} P_{\mathrm{SMR}}$ : p-value from SMR using a second collection of 648 accessions. ${ }^{6} P_{\text {HEIDI }}$ : p-value from HEIDI test using a second collection of 648 accessions.

\begin{tabular}{lcccccc}
\hline Gene & Top SNP $^{1}$ & MAF $^{2}$ & $P_{\text {SMR }}^{3}$ & $P_{\text {HEIDI }}^{4}$ & $P_{\text {SMR }}^{5}$ & $P_{\text {HEIDI }}^{6}$ \\
\hline AT1G11560 & Chr1:3881093 & 0.34 & $6.8 \times 10^{-3}$ & $4.8 \times 10^{-1}$ & $3.2 \times 10^{-2}$ & $2.6 \times 10^{-1}$ \\
AT1G11655 & Chr1:3874970 & 0.39 & $4.1 \times 10^{-2}$ & $9.7 \times 10^{-2}$ & $5.9 \times 10^{-1}$ & NA \\
AT1G11690 & Chr1:4299126 & 0.04 & $3.7 \times 10^{-1}$ & NA & $9.4 \times 10^{-1}$ & NA \\
AT1G11590 & Chr1:3716355 & 0.11 & $5.0 \times 10^{-1}$ & NA & $2.2 \times 10^{-2}$ & $1.5 \times 10^{-1}$ \\
AT1G11482 & Chr1:3830013 & 0.63 & $8.2 \times 10^{-1}$ & NA & $1.5 \times 10^{-1}$ & NA \\
\hline
\end{tabular}

Table 3: Genes in flowering-time pathways whose expression are associated with the detected locus. ${ }^{1}$ p-value from a expression dataset generated from 648 accessions in the $A$. thaliana 1001-genomes project [10. ${ }^{2} \mathrm{FDR}$ value computed from p-value. ${ }^{3}$ Replication p-value from another subset of 140 accessions 14 .

\begin{tabular}{lllll}
\hline Locus ID & Gene Name & p-value $^{1}$ & q-value $^{2}$ & Replication p-value $^{3}$ \\
\hline AT1G17590 & NF-YA8 & $1.6 \times 10^{-7}$ & $2.3 \times 10^{-5}$ & $1.7 \times 10^{-2}$ \\
AT5G53360 & AT5G53360 & $5.8 \times 10^{-7}$ & $5.7 \times 10^{-5}$ & $3.2 \times 10^{-4}$ \\
AT3G57920 & SPL15 & $7.9 \times 10^{-4}$ & $7.8 \times 10^{-3}$ & $1.7 \times 10^{-2}$ \\
AT5G62165 & AGL42 & $1.2 \times 10^{-3}$ & $1.1 \times 10^{-2}$ & $6.3 \times 10^{-3}$ \\
AT5G10140 & FLC & $1.5 \times 10^{-3}$ & $1.3 \times 10^{-2}$ & $5.7 \times 10^{-4}$ \\
AT2G45660 & AGL20 & $1.8 \times 10^{-3}$ & $1.4 \times 10^{-2}$ & $1.2 \times 10^{-3}$ \\
\hline
\end{tabular}

\section{Figures}




\section{Submitted Manuscript}
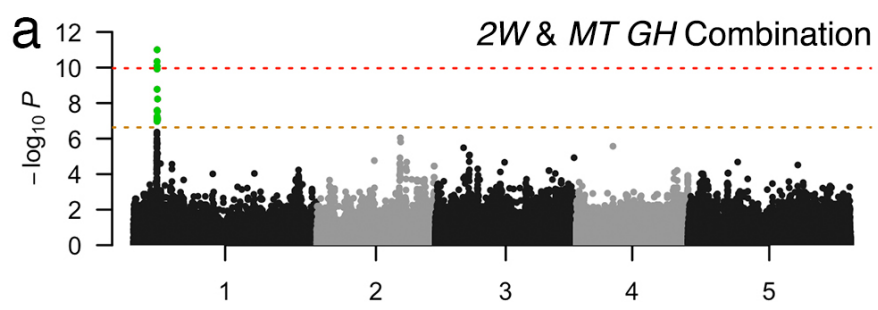

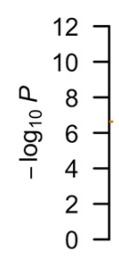
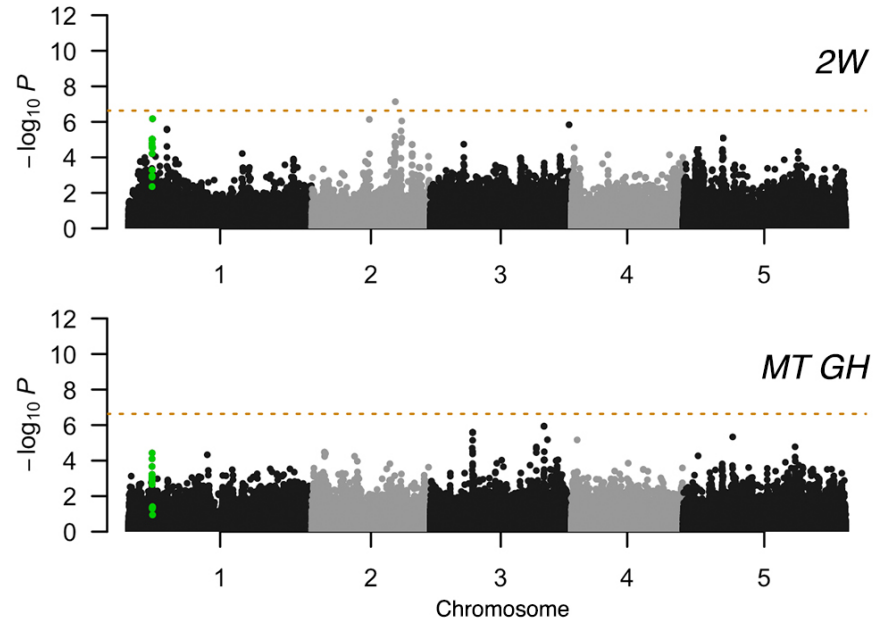

b

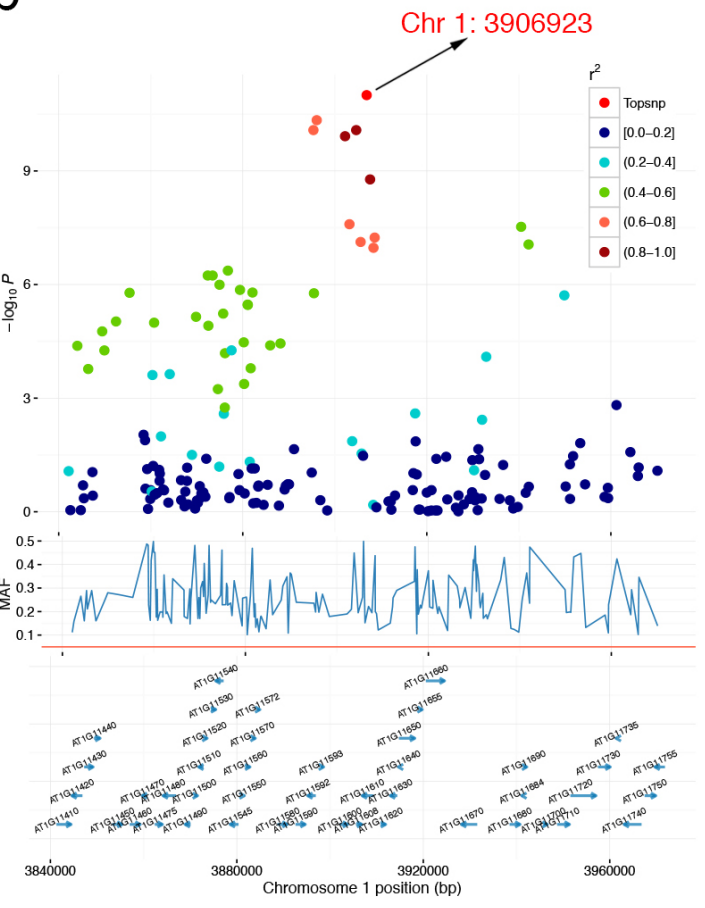

Figure 1: Bivariate genome-wide association analysis of two developmental trait. 2W: Days to flowering time (FT) under long day (LD) with vernalized for $2 \quad{ }^{262}$ wks at $5^{\circ} \mathrm{C}, 8 \mathrm{hrs}$ daylight, MT GH: Maturation period. (a) Manhattan plots comparison of bivariate and univariate analysis results, where the novel variants only discoverable when combining two phenotypes are shown in green. The horizontal dashed line represents a $5 \%$ Bonferroni-corrected genome-wide significant threshold for the number of variants and also the number of tested trait pairs, respectively. (b) Zooming in the novel locus detected using bivariate analysis. r: linkage disequilibrium measured as correlation coefficient between the top variant and each variant in the region. 


\section{Submitted Manuscript}

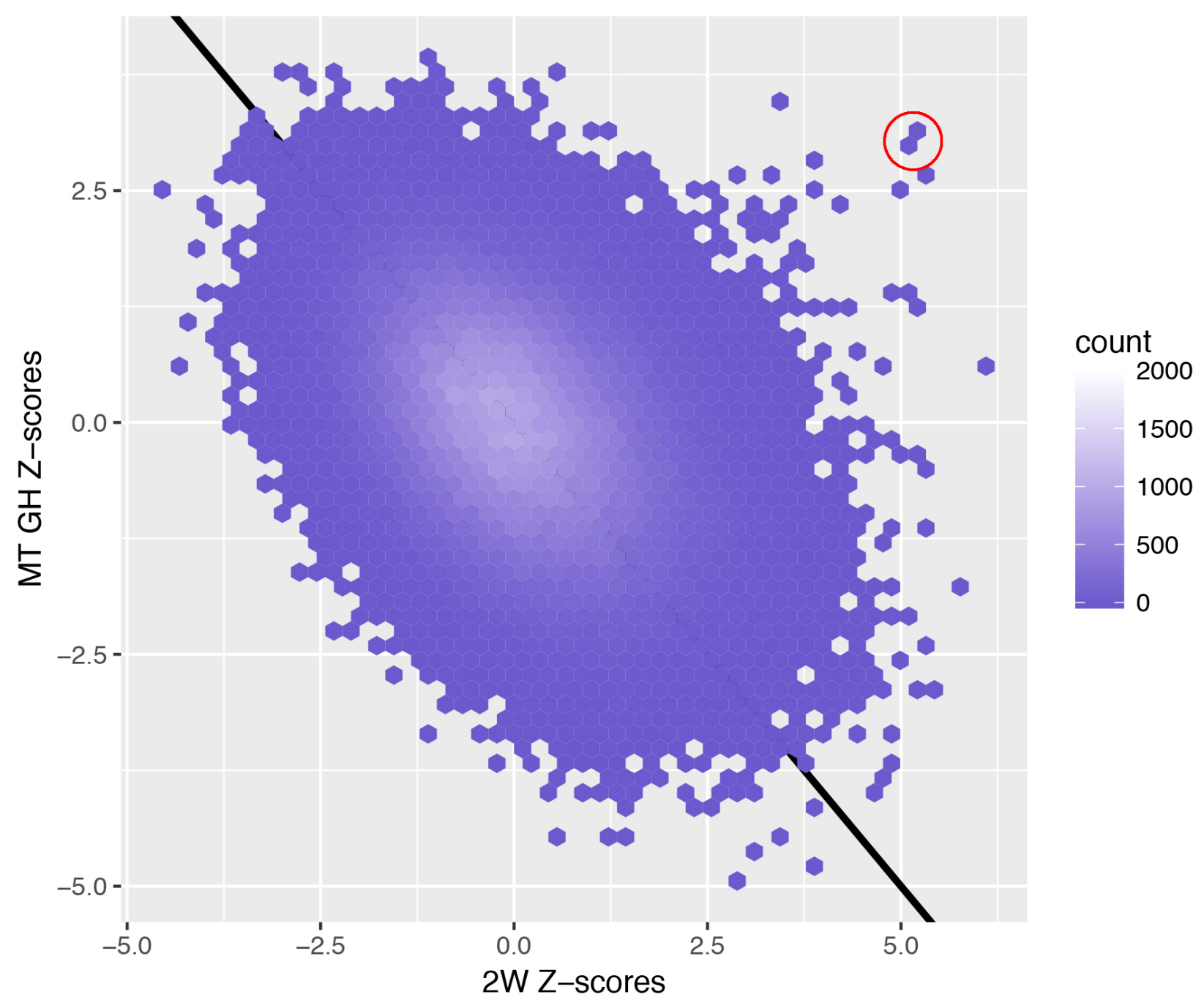

Figure 2: Hexbin scatter plot comparing all Z-scores of the two traits across the genome, showing the bivariate statistical significance of the detected locus. The top variants of the locus is marked on the edge of the empirical bivariate normal distribution with a red circle. The black line with a slope of -1 is provided as a visual guide. 


\section{Submitted Manuscript}

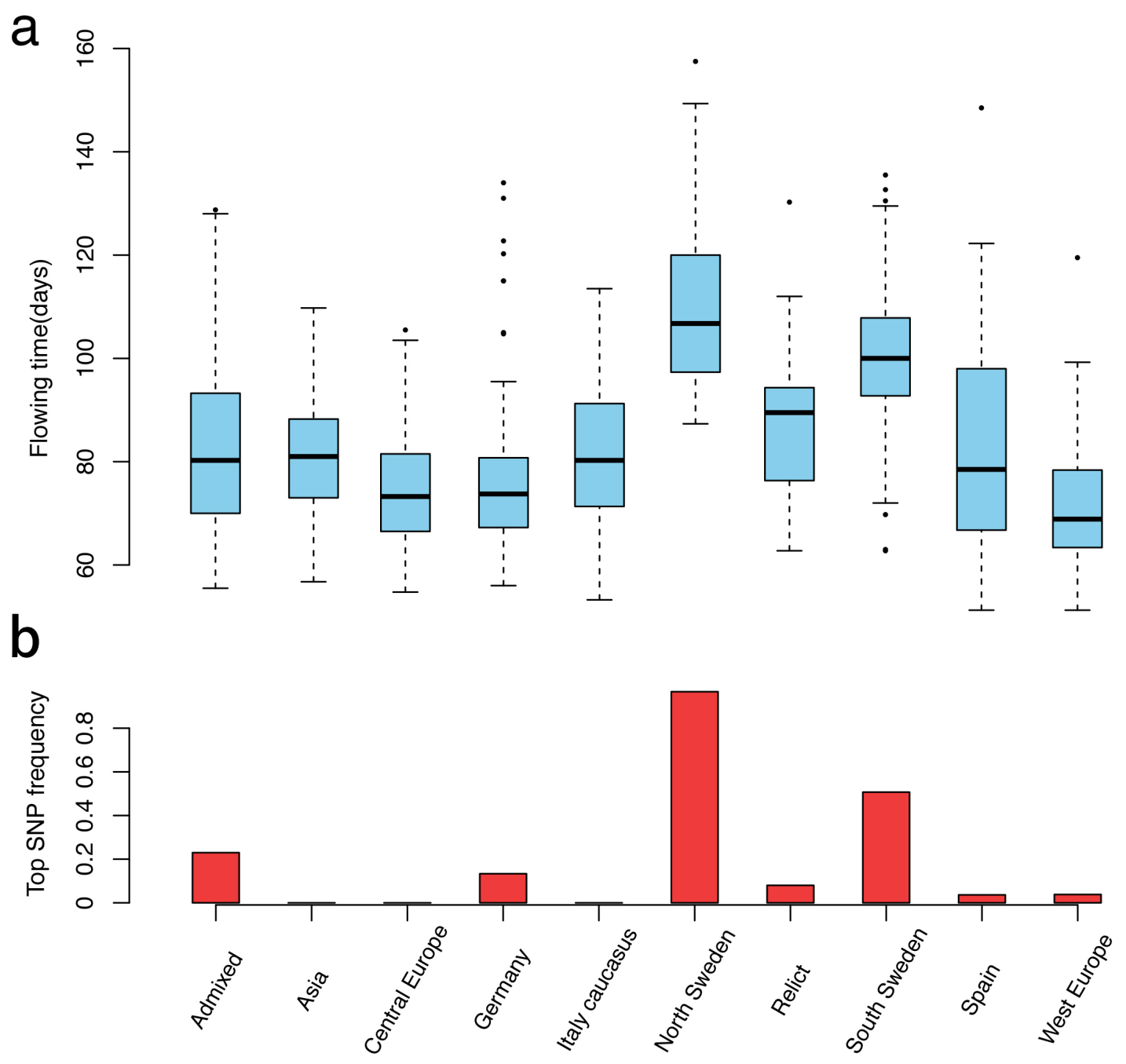

Figure 3: The discovered locus is highly confounded with population

structure. a) Flowering time variation $\left(10^{\circ} \mathrm{C}\right)$ among different sub-populations of Arabidopsis thaliana. These populations are divided by admixture analysis [9]; b) Frequency of the top associated SNP at chromosome 1, 3,906,923 bp in different sub-populations. The association between the structure of the phenotype and that of the allele frequency shows the population confounding at this locus. 


\section{Submitted Manuscript}

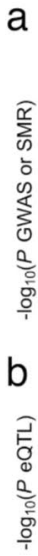

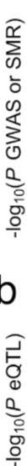

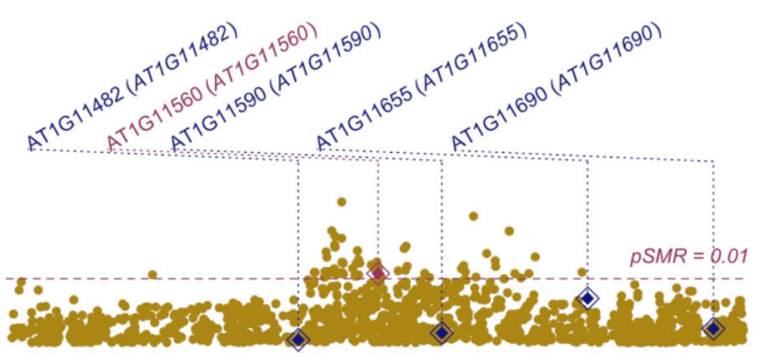

C

Population for GWA Analysis
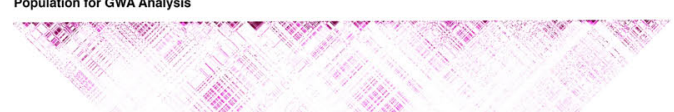

$-7$

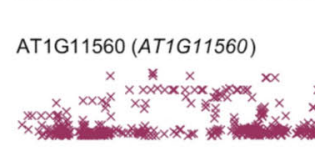

$x^{x}$

$$
\begin{aligned}
& \text { AT1G11482 } \\
& \text { AT1G11560 } \\
& \text { AT1G11590 }
\end{aligned}
$$

\begin{tabular}{|c|c|c|c|c|c|c|}
\hline Г & $T$ & $T$ & $T$ & $T$ & $T$ & ᄀ \\
\hline 3.82 & 3.84 & 3.86 & 3.88 & 3.90 & 3.92 & 3.94 \\
\hline
\end{tabular}

AT1G11655

AT1G11690

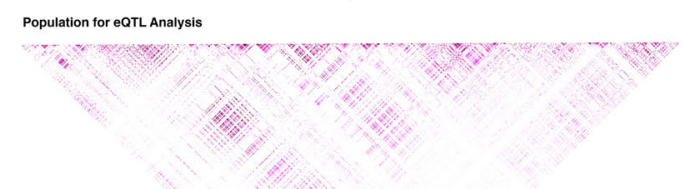

Figure 4: Prioritized candidate genes at the detected locus for flowering

time using SMR analysis. a) Manhattan plot of association between flowering

time at $10^{\circ} \mathrm{C}$ and SNPs around $40 \mathrm{~kb}$ of top associated SNP in bivariate analysis. The

diamonds highlight top eQTL for individual genes; b) Manhattan plot of association between expression of AT1G11560 and SNPs around 40kb of top associated SNP in bivariate analysis. Genes tested in SMR analysis are highlighted using arrows; c) 


\section{MATERIALS \& METHODS}

Genome-wide 250k SNP array genotype data and phenotype data for 199 natural Arabidopsis thaliana accessions

We downloaded a public dataset on collection of 199 natural Arabidopsis thaliana inbred lines contains 107 phenotypes and corresponding genotypes 2 . Those files are publicly available at https://github.com/Gregor-Mendel-Institute/atpolydb/blob/ master/miscellaneous_data/phenotype_published_raw.tsv, and https://github.com/Gregor-Mendel-Institute/atpolydb/blob/master/250k_ snp_data/call_method_75.tar.gz. 214,051 SNPs were available. After filtering out the variants with minor allele frequency less than 0.10, 173,220 SNPs remained.

\section{1,135 natural $A$. thaliana accessions}

1,135 natural Arabidopsis thaliana accessions have been collected and sequenced for the whole genome and transcriptome 9,10 . We downloaded this sequencing dataset and removed the accessions with no measured phenotype and SNPs with minor allele frequency below 0.05 and a call-rate below 0.95. The final dataset includes 1001 individuals with $2,222,379$ SNPs and measured flowing time at $10^{\circ} \mathrm{C}$. To scan for candidate genes, we also downloaded the transcriptome dataset of a subset of this collection $(n=728)$ 10. The final eQTL scan dataset contains RNA-seq derived RPKM-values for 24,150 genes in 648 accessions whose phenotypic and genotypic data are both available.

\section{Whole genome re-sequencing derived SNP genotype and}

RNA-sequencing derived transcriptome data for a population of 144

\section{natural $A$. thaliana accessions}

In an earlier study, Schmitz et al. 14] RNA-sequenced a collection of 144 natural $A$. thaliana accessions. We downloaded this data together with their corresponding whole-genome SNP genotypes available as a part of the 1001 Genomes project 9,10 to replicate our SMR findings. Following the quality control procedure in [18, we removed 
two accessions from the data (Alst_1 and Ws_2) due to missing genotype data and two accessions (Ann_1 and Got_7) due to their low transcript call rate (16,861 and 18,693 genes with transcripts as compared to the range of 22,574 to 26,967 for the other the accessions). The final dataset used for eQTL mapping included 1,347,036 SNPs with MAF above 0.05 and call-rate above 0.95 for 140 accessions, and corresponding RNA-seq derived FPKM-values for 33,554 genes.

\section{Single-trait analysis for flowering time trait}

For all available traits in this dataset, we first performed a mixed model based single trait genome wide association analysis to generate single trait summaries statistics. Those summaries statistics were used as input for double trait analysis described in the following section. To replicate our signal, we also performed a single trait genome wide association analysis using a collection generated in 1001-genomes project 9 . To correct for the population structure in these $A$. thaliana accessions, single-trait genome wide scan was performed based on linear mixed models, using the polygenic and mmscore procedure in GenABEL 23.

\section{Double-trait genome-wide association analysis}

We performed double-trait genome scans using our recently developed multivariate analysis method implemented in the MultiABEL package [15]. The method takes the whole-genome summary statistics to infer phenotypic correlation coefficients and conducts MANOVA analysis. The phenotypic correlation coefficient of two traits can be unbiasedly estimated by the correlation of genome-wide Z-scores, which is proportional to the phenotypic correlation on the liability scale. In this way, the bivariate MANOVA analysis is carried out on the liability scale, with bivariate p-values reported.

\section{eQTL and SMR analysis}

We screened for candidate genes by analyzing the expression data in a subset of the 1001-genomes collection containing 140 accessions. Expression values for 19 genes around $20 \mathrm{~kb}$ up/downstream of the top associated SNP were extracted from [14. 14 genes did not pass Kolmogorov-Smirnov test (ks test statistics ; 0.8) were filtered out 
due to potential unreliable measurement mentioned in [18]. The remaining five genes were subsequently passed onto eQTL mapping using qtscore procedure in

GenABEL 23]. Output were reformatted according to the description in [19]. Together with the flowering time single-trait scan results 9, these were further passed onto SMR analysis scanning for association between individual gene expression and flowering time. The SMR analysis were repeated for 5 top candidates, in an independent gene expression dataset containing 648 accessions 10 following the same procedure.

345

\section{References}

1. Crawley MJ. Plant Ecology. John Wiley \& Sons; 2009.

2. Atwell S, Huang YS, Vilhjálmsson BJ, Willems G, Horton M, Li Y, et al. Genome-wide association study of 107 phenotypes in Arabidopsis thaliana inbred lines. Nature. 2010;465(7298):627-631.

3. Hirschhorn JN, Daly MJ. Genome-wide association studies for common diseases and complex traits. Nature reviews Genetics. 2005;6(2):95-108.

4. Huang X, Wei X, Sang T, Zhao Q, Feng Q, Zhao Y, et al. Genome-wide association studies of 14 agronomic traits in rice landraces. Nature genetics. 2010;42(11):961-967.

5. Yang J, Benyamin B, McEvoy BP, Gordon S, Henders AK, Nyholt DR, et al. 362 Common SNPs explain a large proportion of the heritability for human height. Nature genetics. 2010;42(7):565-569.

6. Korte A, Farlow A. The advantages and limitations of trait analysis with GWAS: 365 a review. Plant methods. 2013;9:29.

7. Yang J, Zaitlen NA, Goddard ME, Visscher PM, Price AL. Advantages and pitfalls in the application of mixed-model association methods. Nature genetics. 2014;46(2):100-106.

8. Wellenreuther M, Hansson B. Detecting Polygenic Evolution: Problems, Pitfalls, 370 and Promises. Trends in genetics : TIG. 2016;32(3):155-164. 
9. Consortium G. 1,135 Genomes Reveal the Global Pattern of Polymorphism in Arabidopsis thaliana. Cell. 2016;166(2):481-491.

10. Kawakatsu T, Huang SSC, Jupe F, Sasaki E, Schmitz RJ, Urich MA, et al. Epigenomic Diversity in a Global Collection of Arabidopsis thaliana Accessions. Cell. 2016;166(2):492-505.

11. Shen X, Pettersson M, Rönnegård L, Carlborg O. Inheritance beyond plain heritability: variance-controlling genes in Arabidopsis thaliana. PLoS genetics. 2012;8(8):e1002839.

372

373

374

375

376

377

378

379

12. Wang B, Li Z, Xu W, Feng X, Wan Q, Zan Y, et al. Bivariate genomic analysis identifies a hidden locus associated with bacteria hypersensitive response in Arabidopsis thaliana. Scientific reports. 2017;7:45281.

13. Shen X, De Jonge J, Forsberg SKG, Pettersson ME, Sheng Z, Hennig L, et al. Natural CMT2 variation is associated with genome-wide methylation changes and temperature seasonality. PLoS genetics. 2014;10(12):e1004842.

14. Schmitz RJ, Schultz MD, Urich MA, Nery JR, Pelizzola M, Libiger O, et al. Patterns of population epigenomic diversity. Nature. 2013;495(7440):193-198.

15. Shen X, Klarić L, Sharapov S, Mangino M, Ning Z, Wu D, et al. Multivariate discovery and replication of five novel loci associated with Immunoglobulin G N-glycosylation. Nature communications. 2017;8(1):447.

16. Alexander DH, Novembre J, Lange K. Fast model-based estimation of ancestry in unrelated individuals. Genome research. 2009;19(9):1655-1664.

17. Dittmar EL, Oakley CG, Ågren J, Schemske DW. Flowering time QTL in natural populations of Arabidopsis thaliana and implications for their adaptive value. Molecular ecology. 2014;23(17):4291-4303.

18. Zan Y, Shen X, Forsberg SKG, Carlborg O. Genetic Regulation of Transcriptional Variation in Natural Arabidopsis thaliana Accessions. G3 (Bethesda, Md). 2016;6(8):2319-2328.
380 
19. Zhu Z, Zhang F, Hu H, Bakshi A, Robinson MR, Powell JE, et al. Integration of 399 summary data from GWAS and eQTL studies predicts complex trait gene targets. $\quad 400$ Nature genetics. 2016;48(5):481-487.

20. Burgess S, Scott RA, Timpson NJ, Davey Smith G, Thompson SG, EPICInterAct Consortium. Using published data in Mendelian randomization: a blueprint for efficient identification of causal risk factors. European journal of epidemiology. 2015;30:543.

21. Visscher PM, Yang J. A plethora of pleiotropy across complex traits. Nature genetics. 2016;48(7):707-708.

22. Brachi B, Faure N, Horton M, Flahauw E, Vazquez A, Nordborg M, et al. Linkage and association mapping of Arabidopsis thaliana flowering time in nature. $\quad{ }^{409}$ PLoS genetics. 2010;6(5):e1000940.

23. Aulchenko YS, Ripke S, Isaacs A, van Duijn CM. GenABEL: an R library for 411 genome-wide association analysis. Bioinformatics (Oxford, England). 
Table S1: Phenotypes included in the bivariate analyses. Details about

phenotyping can be referred to [2].

\begin{tabular}{|c|c|c|}
\hline Phenotype & Description & Number of Accessions \\
\hline LD & Days to flowering time (FT) under Long Day (LD) & 167 \\
\hline LDV & Days to flowering time (FT) under Long Day (LD) (5 wks vernalization) & 168 \\
\hline SD & Days to flowering time (FT) under Short Day (SD) & 162 \\
\hline SDV & Days to flowering time (FT) under Short Day (SD) (5 wks vernalization) & 159 \\
\hline oW & Days to FT under LD without vernalization & 137 \\
\hline $2 \mathrm{~W}$ & Days to FT under LD with 2 wks vernalization & 152 \\
\hline $4 \mathrm{~W}$ & Days to FT under LD with 4 wks vernalization & 119 \\
\hline $8 \mathrm{~W}$ & Days to FT under LD with 8wks vernalization & 155 \\
\hline FLC & FLC gene expression & 167 \\
\hline FRI & FRI gene expression & 164 \\
\hline FT10 & Flowering time $(\mathrm{FT}), 10^{\circ} \mathrm{C}$ & 194 \\
\hline FT16 & Flowering time $(\mathrm{FT}), 16^{\circ} \mathrm{C}$ & 193 \\
\hline FT22 & Flowering time $(\mathrm{FT}), 22^{\circ} \mathrm{C}$ & 193 \\
\hline LN10 & leaf number at flowering time $(\mathrm{LN}), 10^{\circ} \mathrm{C}$ & 177 \\
\hline LN16 & leaf number at flowering time $(\mathrm{LN}), 16^{\circ} \mathrm{C}$ & 176 \\
\hline $\mathrm{LN} 22$ & leaf number at flowering time $(\mathrm{LN}), 22^{\circ} \mathrm{C}$ & 176 \\
\hline $8 \mathrm{~W}$ GH FT & Days to FT with 8 wks vernalization & 162 \\
\hline $8 \mathrm{~W}$ GH LN & LN at FT with 8 wks vernalization & 163 \\
\hline 0W GH FT & Days to FT without vernalization & 153 \\
\hline 0W GH LN & LN at FT without vernalization & 135 \\
\hline FT Field & Days to flowering of plants grown in the field & 180 \\
\hline FT Diameter Field & Plant diameter at flowering (field) & 180 \\
\hline FT GH & Days to flowering (greenhouse) & 166 \\
\hline LES & Presence or absence of lesioning & 95 \\
\hline YEL & Presence or absence of yellowing & 95 \\
\hline LY & Presence or absence of either lesioning or yellowing & 95 \\
\hline FW & Fresh weight of plants & 95 \\
\hline DW & Dry weight of plants & 95 \\
\hline Chlorosis 10 & Visual chlorosis presence, $10^{\circ} \mathrm{C}$ & 177 \\
\hline Chlorosis 16 & Visual chlorosis presence, $16^{\circ} \mathrm{C}$ & 176 \\
\hline Chlorosis 22 & Visual chlorosis presence, $22^{\circ} \mathrm{C}$ & 176 \\
\hline
\end{tabular}


Anthocyanin 10

Anthocyanin 16

Anthocyanin 22

Seed Dormancy

Germ 10

Germ 16

Germ 22

Seedling Growth

Vern Growth

After Vern Growth

Secondary Dormancy

Germ in dark

DSDS50

Seed bank 133-91

Storage 7 days

Storage 28 days

Storage 56 days

Hypocotyl length

Width 10

Width 16

Width 22

Leaf serr 10

Leaf serr 16

Leaf serr 22

Leaf roll 10

Leaf roll 16

Leaf roll 22

Rosette Erect 22

Silique 16

Silique 22

FT Duration GH

LC Duration GH

LFS GH

MT GH

RP GH
Visual anthocyanin presence, $10^{\circ} \mathrm{C}$

177

Visual anthocyanin presence, $16^{\circ} \mathrm{C}$

176

Visual anthocyanin presence, $22^{\circ} \mathrm{C}$

177

Seed dormancy level

83

Days to germination, $10^{\circ} \mathrm{C}$

177

Days to germination, $16^{\circ} \mathrm{C}$

176

Days to germination, $22^{\circ} \mathrm{C}$

177

Seedling growth rate

100

Vegetative growth rate during vernalization

110

Vegetative growth rate after vernalization

110

Decrease in germination rate after prolonged exposure to cold temperature

93

Germination in the dark

93

Duration of seed dry storage required for $50 \%$ of the seeds to germinate

109

Non-monotonous dynamic of dormancy release

110

Primary dormancy, 7 days dry storage

110

Primary dormancy, 28 days dry storage

110

Primary dormancy, 56 days dry storage

110

Hypocotyl length

89

Plant diameter, $10^{\circ} \mathrm{C}$

176

Plant diameter, $16^{\circ} \mathrm{C}$

175

Plant diameter, $22^{\circ} \mathrm{C}$

175

Level of leaf serration, $10^{\circ} \mathrm{C}$

174

Level of leaf serration, $16^{\circ} \mathrm{C}$

176

Level of leaf serration, $22^{\circ} \mathrm{C}$

176

Leaf roll presence, $10^{\circ} \mathrm{C}$

177

Leaf roll presence, $16^{\circ} \mathrm{C}$

176

Leaf roll presence, $22^{\circ} \mathrm{C}$

176

Presence of rosette errectness, $22^{\circ} \mathrm{C}$

176

Silique length, $16^{\circ} \mathrm{C}$

Silique length, $22^{\circ} \mathrm{C}$

Flowering period duration

147

Life cycle period

147

Last flower senescence

148

Maturation period

147

Reproduction period 


\section{Submitted Manuscript}

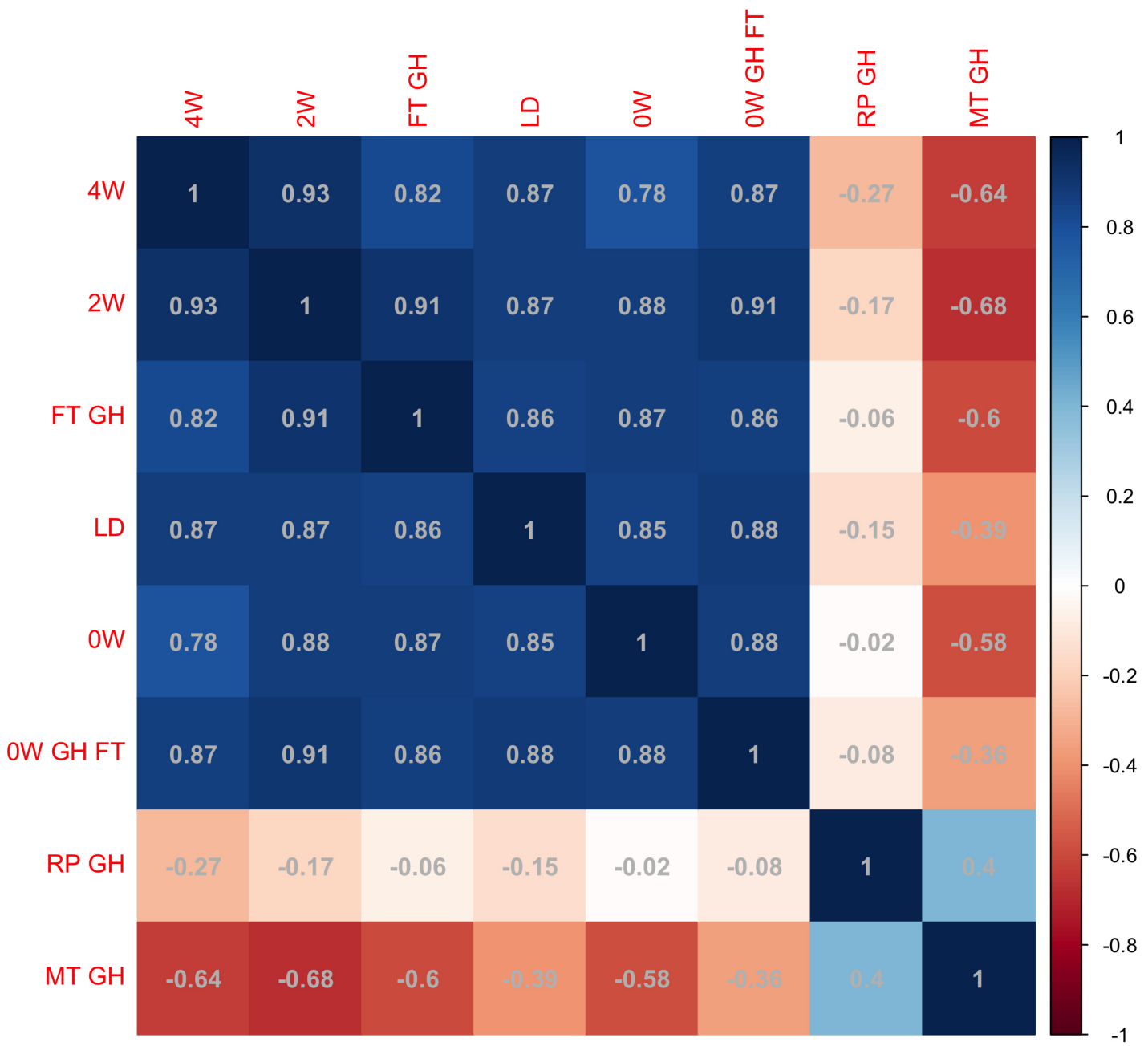

Figure S1: Phenotypic correlations among flowering time related traits, maturation period and reproduction period phenotypes. The flowering time related traits are: 4W: Days to flowering time (FT) under long day (LD) with vernalized for 4 wks at $5^{\circ} \mathrm{C}, 8 \mathrm{hrs}$ daylight; $2 \mathrm{~W}$ : Days to flowering time (FT) under long day (LD) with vernalized for 2 wks at $5{ }^{\circ} \mathrm{C}, 8 \mathrm{hrs}$ daylight; FT GH: Days to flowering (greenhouse); LD: Days to flowering time (FT) under Long Day (LD); 0W: Days to flowering time (FT) under Long Day (LD) without vernalization; 0W GH FT: Days to flowering time (FT). 


\section{Submitted Manuscript}

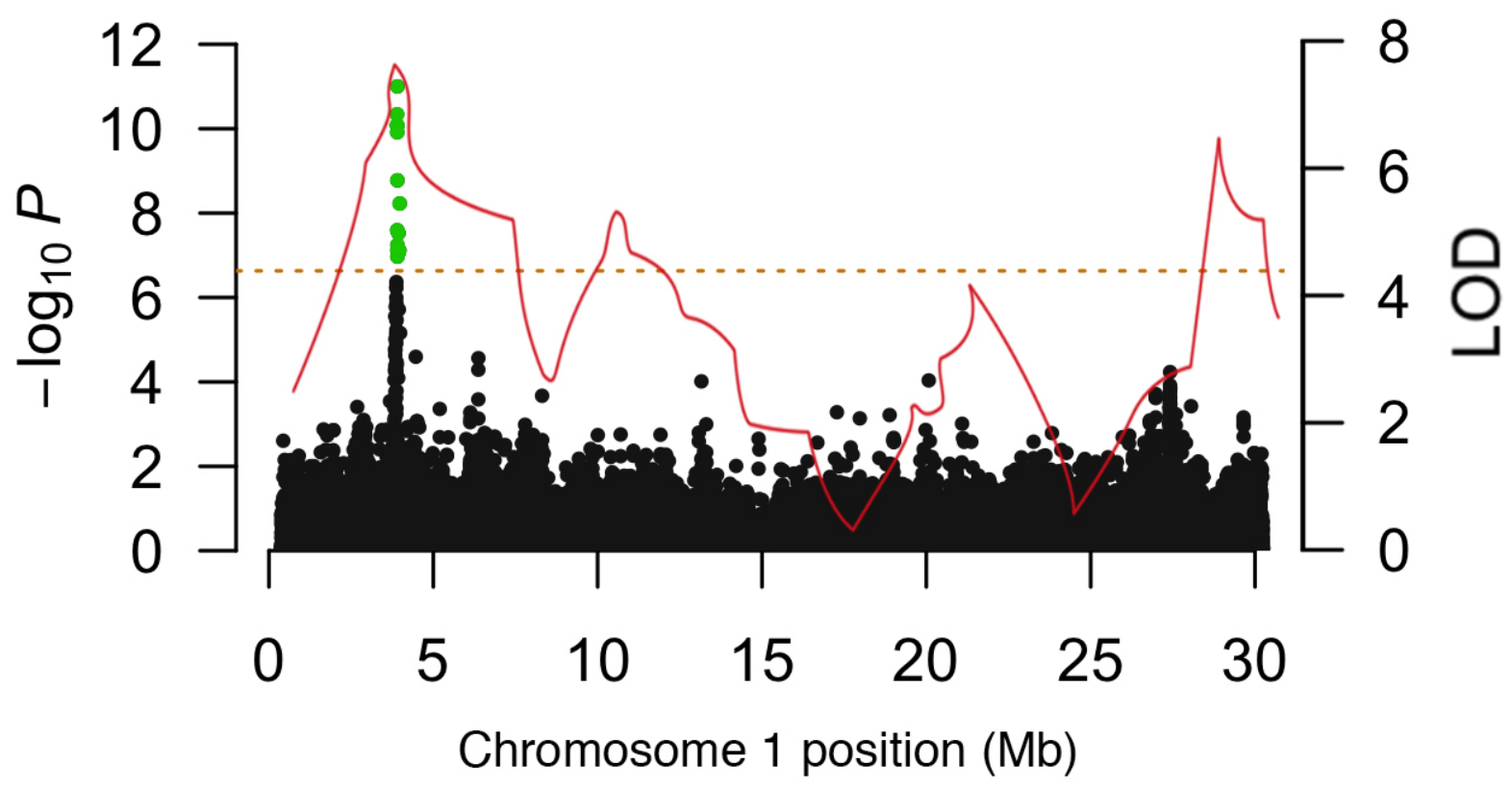

Figure S2: Overlapping between QTL mapping and double-trait GWAS result. The curve shows stepwise LOD profiles in chromosome 1 that are generated from a QTL mapping study using a cross between Italy and Sweden population analyzed by [17] (reproduced by depicting the curvature of Figure 3a therein). The Manhattan plot shows chromosome 1 signal in our bivariate analysis. 


\section{Submitted Manuscript}

$$
\left.\begin{array}{rr}
a & 10 \\
& 8 \\
0 & \\
0 & 6 \\
0 & \\
\frac{8}{1} & 4 \\
& 2 \\
& 0
\end{array}\right]
$$

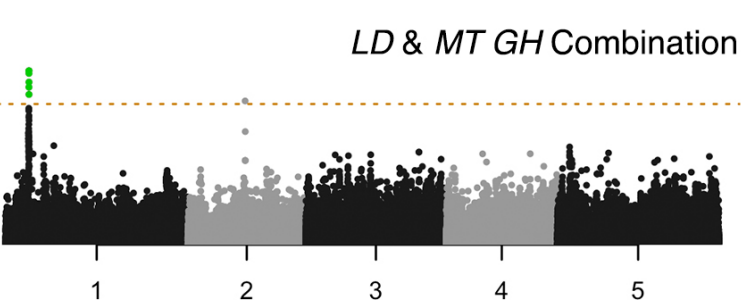

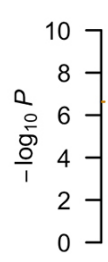
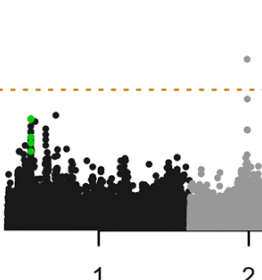

\section{$L D$}
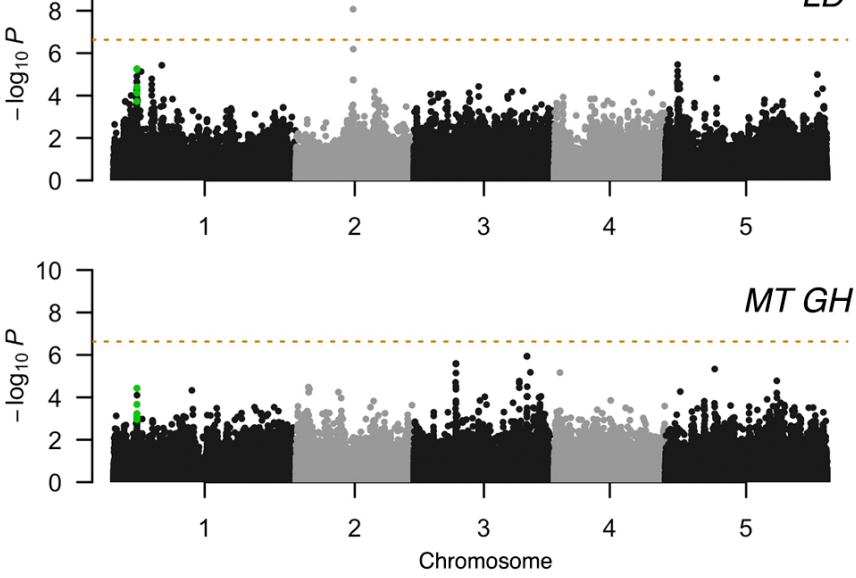

b
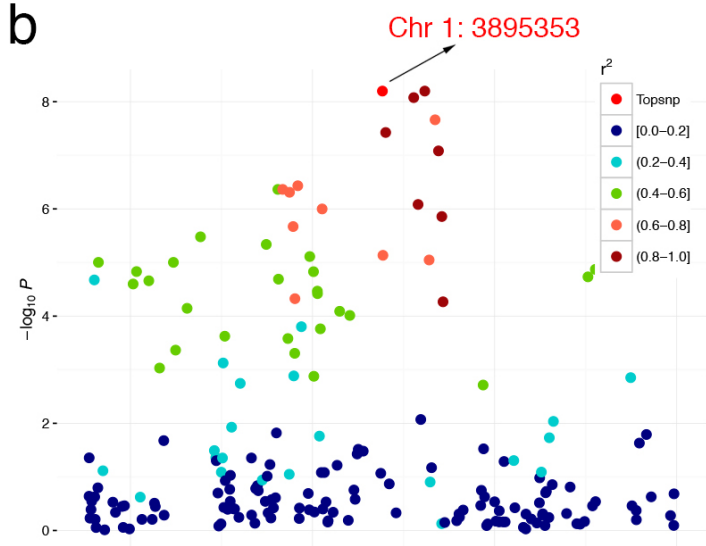

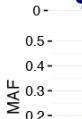

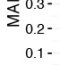

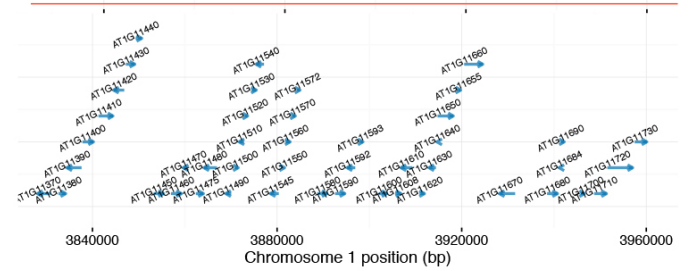

Figure S3: Bivariate genome-wide association analysis of two developmental trait, LD: Days to flowering time (FT) under Long Day (LD), MT GH: Maturation period. (a) Manhattan plots comparison of bivariate and univariate analysis results, where the novel variants only discoverable when combining two phenotypes are shown in green. The horizontal dashed line represents a 5\% Bonferroni-corrected genome-wide significant threshold. (b) Zooming in the novel locus detected using bivariate analysis. r: linkage disequilibrium measured as correlation coefficient between the top variant and each variant in the region. 


\section{Submitted Manuscript}

$$
\left.\begin{array}{rr}
a & 10 \\
& 8 \\
0 & \\
0 & 6 \\
0 & 0 \\
\hline & 4 \\
1 & 2 \\
& 2 \\
& 0
\end{array}\right]
$$

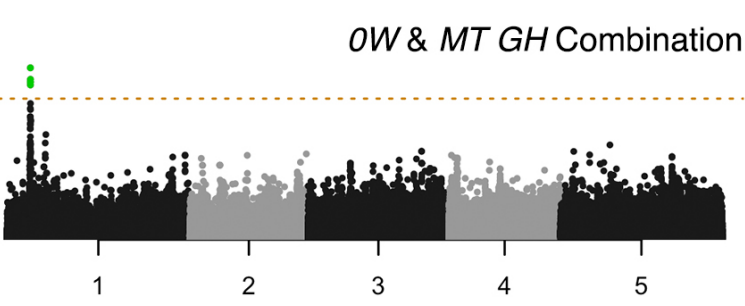

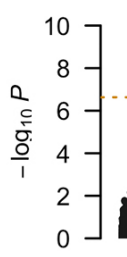

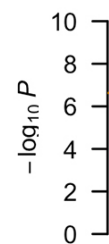

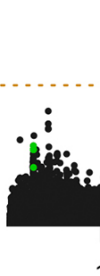

2

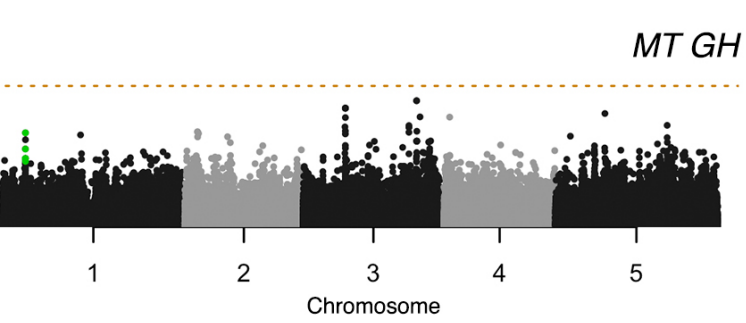

OW
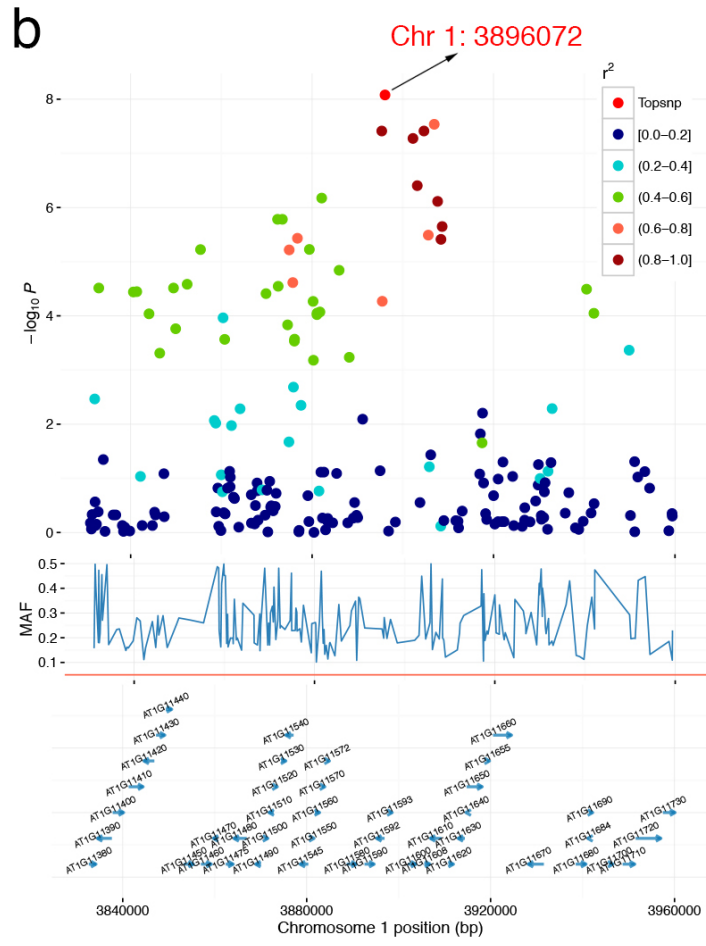

Figure S4: Bivariate genome-wide association analysis of two developmental trait, 0W: Days to flowering time (FT) under Long Day (LD) without vernalization, MT GH: Maturation period. (a) Manhattan plots comparison of bivariate and univariate analysis results, where the novel variants only discoverable when combining two phenotypes are shown in green. The horizontal dashed line represents a $5 \%$ Bonferroni-corrected genome-wide significant threshold. (b) Zooming in the novel locus detected using bivariate analysis. r: linkage disequilibrium measured as correlation coefficient between the top variant and each variant in the region. 


\section{Submitted Manuscript}
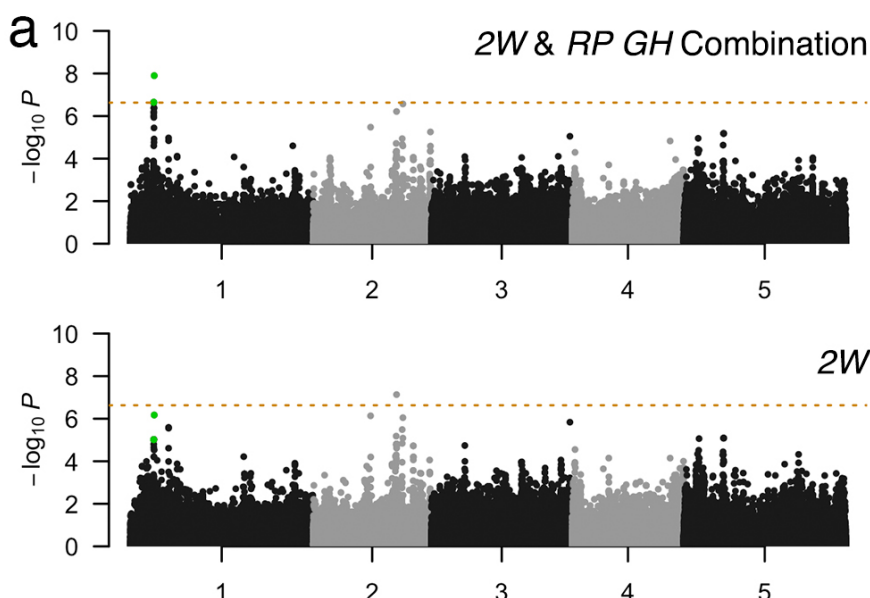

5

$2 W$
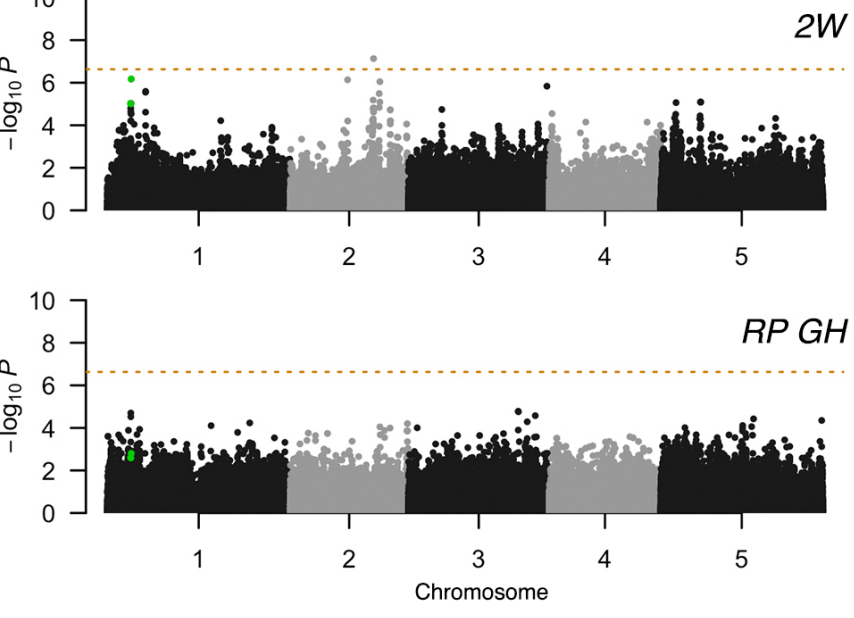
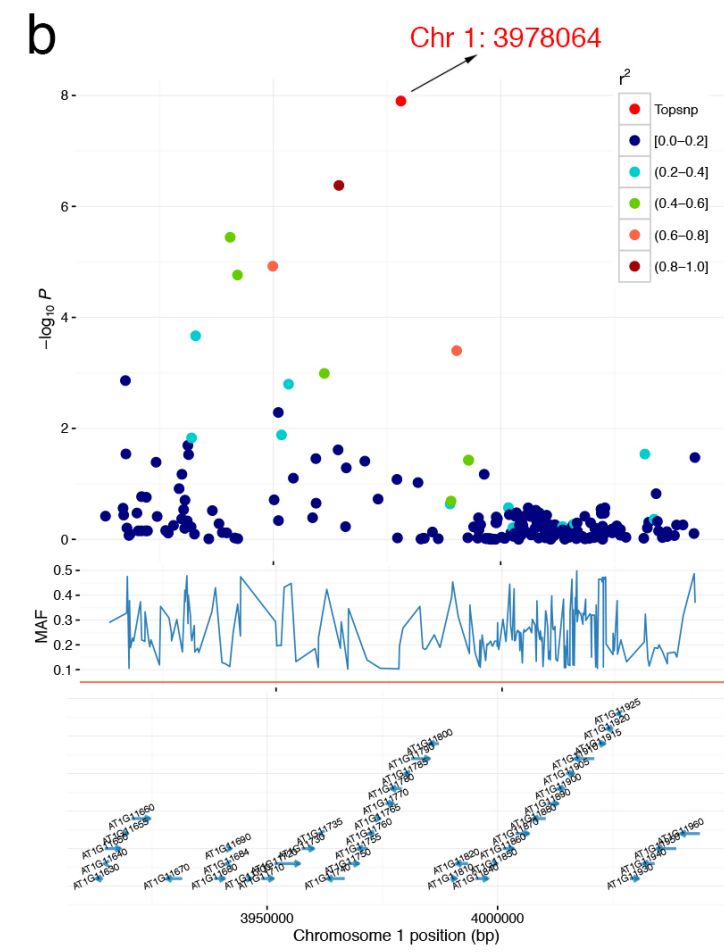

Figure S5: Bivariate genome-wide association analysis of two

developmental trait, 2W: Days to flowering time (FT) under long day (LD) with vernalized for 2 wks at $5^{\circ} \mathrm{C}, 8 \mathrm{hrs}$ daylight, RP GH: Reproduction 449

period. (a) Manhattan plots comparison of bivariate and univariate analysis results, where the novel variants only discoverable when combining two phenotypes are shown in green. The horizontal dashed line represents a $5 \%$ Bonferroni-corrected genome-wide significant threshold. (b) Zooming in the novel locus detected using bivariate analysis. r: linkage disequilibrium measured as correlation coefficient between the top variant and each variant in the region. 


\section{Submitted Manuscript}
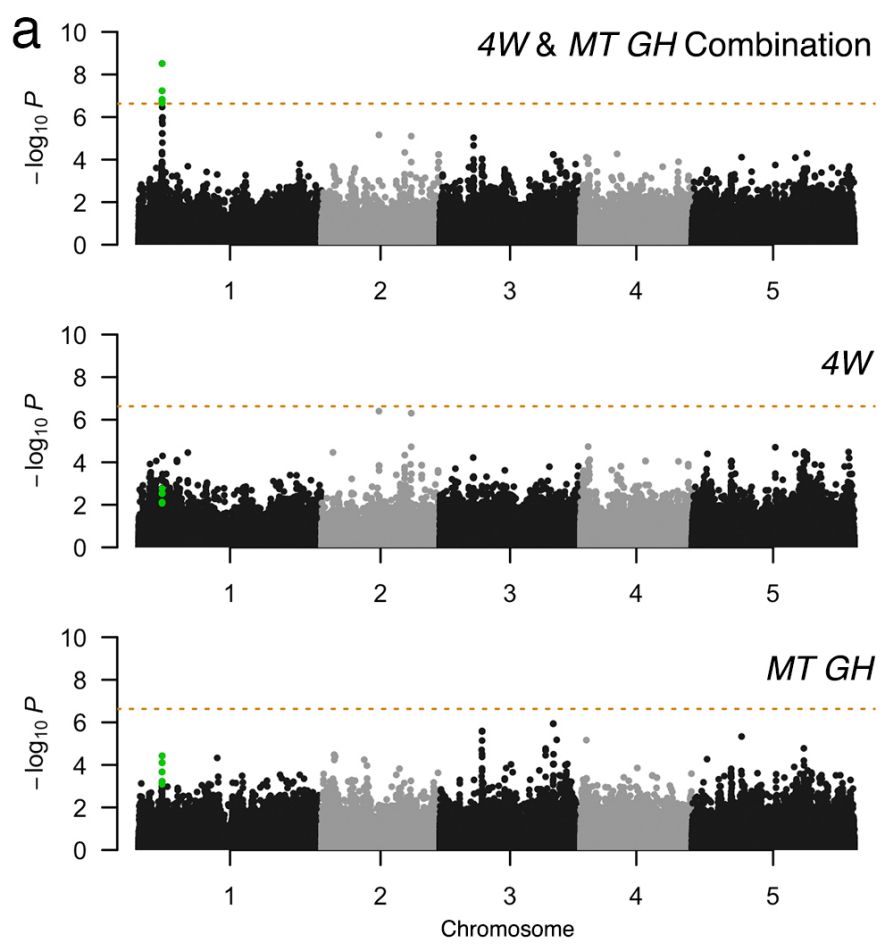

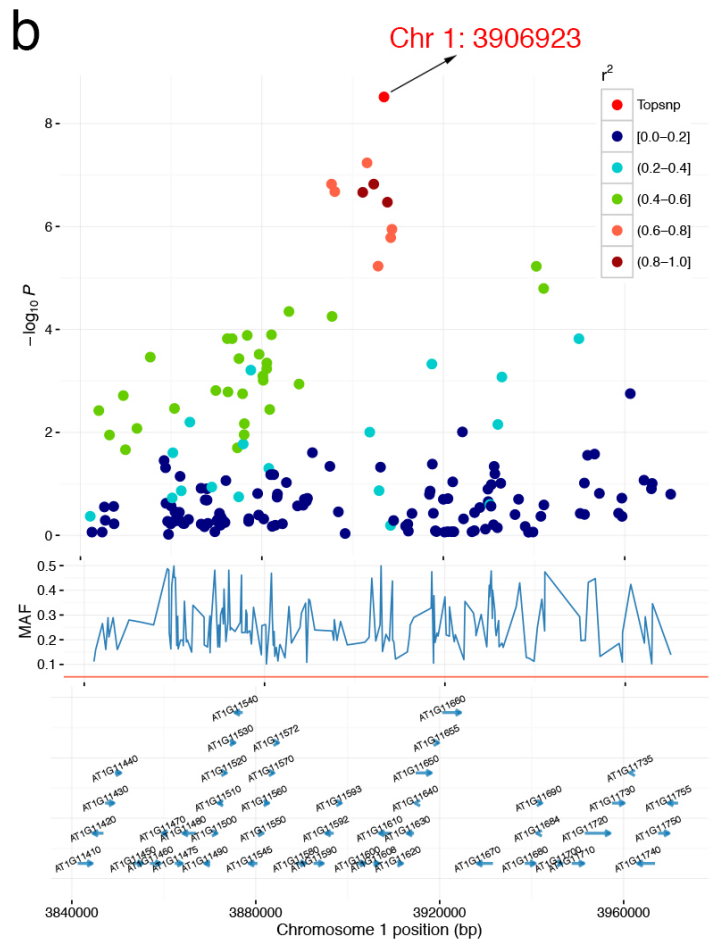

Figure S6: Bivariate genome-wide association analysis of two developmental trait, 4W: Days to flowering time (FT) under long day (LD) with vernalized for 4 wks at $5^{\circ} \mathrm{C}, 8 \mathrm{hrs}$ daylight, MT GH: Maturation

period. (a) Manhattan plots comparison of bivariate and univariate analysis results, where the novel variants only discoverable when combining two phenotypes are shown in green. The horizontal dashed line represents a $5 \%$ Bonferroni-corrected genome-wide significant threshold. (b) Zooming in the novel locus detected using bivariate analysis. r: linkage disequilibrium measured as correlation coefficient between the top variant and each variant in the region. 


\section{Submitted Manuscript}
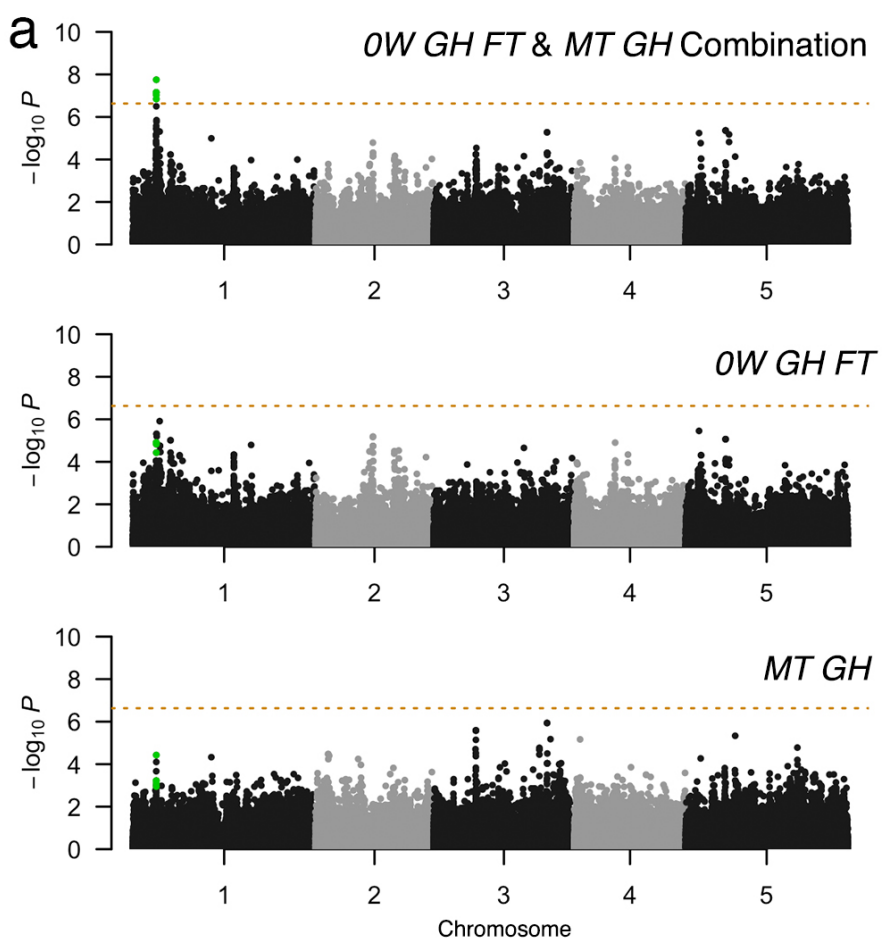

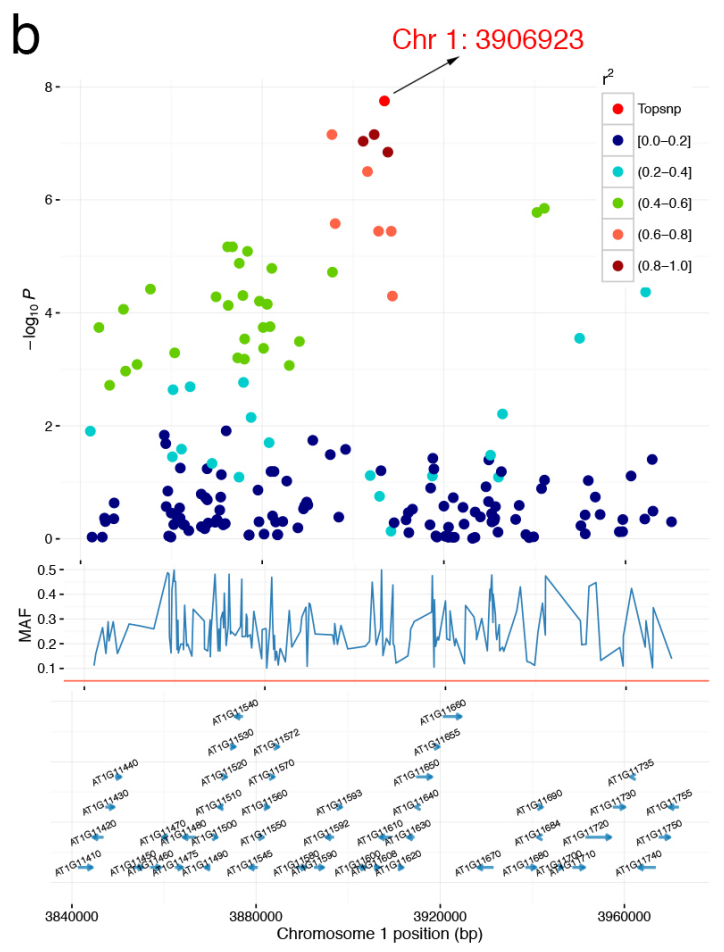

Figure S7: Bivariate genome-wide association analysis of two developmental trait, OW GH FT: Days to flowering time (FT), MT GH:

Maturation period. (a) Manhattan plots comparison of bivariate and univariate analysis results, where the novel variants only discoverable when combining two phenotypes are shown in green. The horizontal dashed line represents a $5 \%$ Bonferroni-corrected genome-wide significant threshold. (b) Zooming in the novel locus detected using bivariate analysis. $r$ : linkage disequilibrium measured as correlation coefficient between the top variant and each variant in the region. 


\section{Submitted Manuscript}
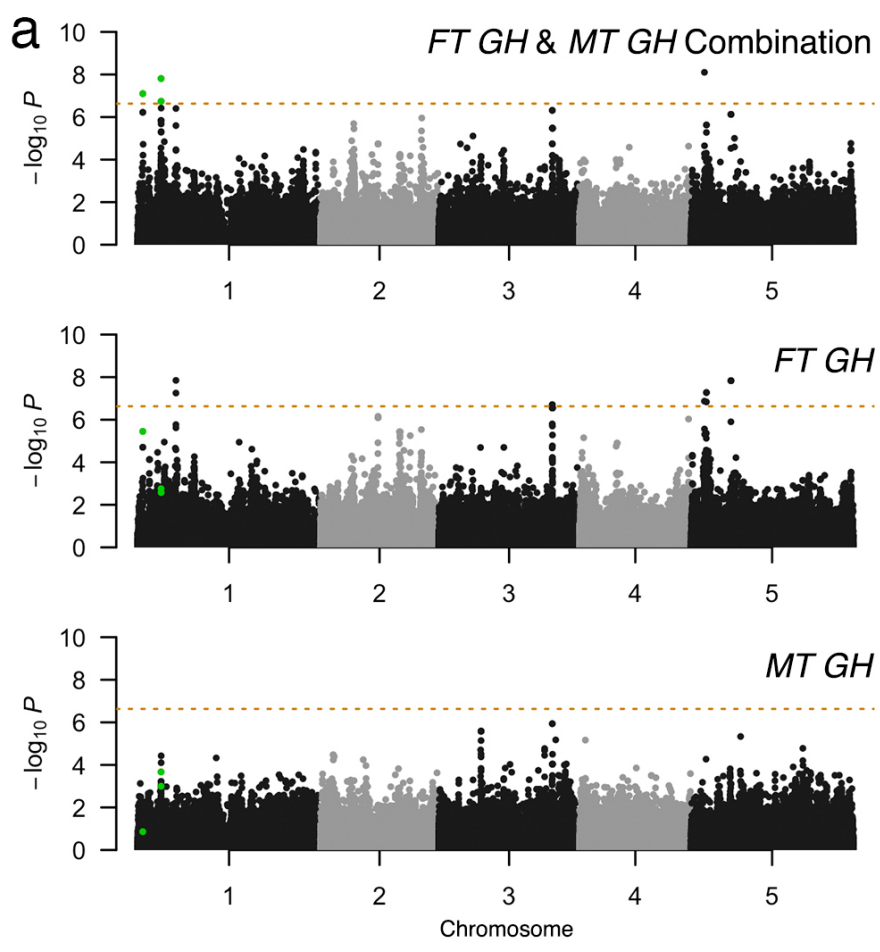

b
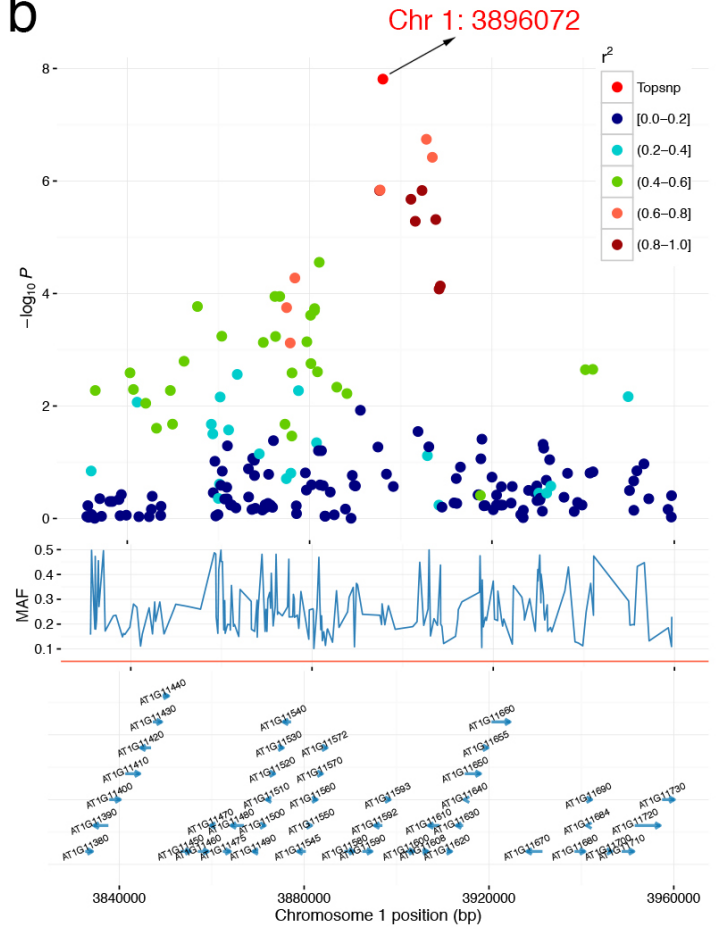

Figure S8: Bivariate genome-wide association analysis of two developmental trait, FT GH: Days to flowering (greenhouse), MT GH:

Maturation period. (a) Manhattan plots comparison of bivariate and univariate analysis results, where the novel variants only discoverable when combining two phenotypes are shown in green. The horizontal dashed line represents a $5 \%$ Bonferroni-corrected genome-wide significant threshold. (b) Zooming in the novel locus detected using bivariate analysis. $r$ : linkage disequilibrium measured as correlation coefficient between the top variant and each variant in the region. 